\title{
Myocardial infarct delineation in vivo using diffusion tensor MRI and the tractographic propagation angle
}

\author{
Choukri Mekkaoui", Shuning Huang, Guangping Dai, Timothy G Reese, Jeremy Ruskin, Udo Hoffmann, \\ Marcel P Jackowski, David E Sosnovik
}

From 16th Annual SCMR Scientific Sessions

San Francisco, CA, USA. 31 January - 3 February 2013

\section{Background}

Delayed gadolinium enhancement (Gd-DE) is widely used to detect scar formation following myocardial infarction (MI) [1], but cannot be performed in patients with renal impairment. Here we use the tractographic propagation angle (PA), a novel index derived from 3D diffusion tensor MRI (DTI), to detect changes in myocardial fiber architecture post-MI [2]. We compare image segmentation based on the tractographic PA to infarct delineation with GdDE.

\section{Methods}

Normal human $(\mathrm{n}=5)$ and infarcted sheep hearts $(\mathrm{n}=6)$ were studied ex vivo. Infarcted mice $(\mathrm{n}=7)$ were imaged in vivo. MI was produced in C57BL6 mice via permanent ligation of the left coronary artery. In vivo DTI was performed on a 9.4T scanner (Bruker) using a 3D fat-suppressed single-shot 3D spin echo EPI sequence with motion-compensated diffusion-encoding gradients in 24 directions. Other parameters were: TR/TE $=2000 / 13.5 \mathrm{~ms}$, b-value $500-700 \mathrm{~s} / \mathrm{mm}^{2}$ and isotropic resolution of 280 $\mu \mathrm{m}$. The human and sheep hearts were imaged on a clinical 3T Siemens scanner with an isotropic resolution of 2 $\mathrm{mm}^{3}, \mathrm{TR} / \mathrm{TE}=8430 / 96 \mathrm{~ms}$, and a b-value of $2000 \mathrm{~s} / \mathrm{mm}^{2}$. The tractographic propagation angle PA was defined as the angle between two adjacent principal eigenvectors $\left(\hat{e}_{i j}\right.$, $\hat{\mathrm{e}}_{\mathrm{ij}+1}$ ) relative to a given fiber (Figure 1A). PA values were computed along myofiber trajectories within the principal eigenvector field using a $4^{\text {th }}$ order Runge-Kutta integration method. Gd-DE imaging was performed 10min after the injection of $0.2 \mathrm{mmol}$ Gd-DTPA $/ \mathrm{kg}$. A short axis slice

\footnotetext{
Radiology, Harvard Medical School - Massachusetts General Hospital, Boston,
} MA, USA

\section{MA, USA}

(c) 2013 Mekkaoui et al; licensee BioMed Central Ltd. This is an Open Access article distributed under the terms of the Creative Commons Attribution License (http://creativecommons.org/licenses/by/2.0), which permits unrestricted use, distribution, and reproduction in any medium, provided the original work is properly cited. through the infarcted myocardium was acquired using a cardiac-gated inversion recovery gradient echo sequence. Infarcted regions were segmented automatically on the Gd-DE images using a threshold of 2 standard deviations above normal. A PA threshold value greater than 4 degrees was used to automatically segment infarcted myocardium. Percent infarct size was calculated with both techniques and correlated.

\section{Results}

Tractography of a normal human heart color-coded by the PA is shown in Figure 1B. PA in the normal myocardium is highly homogeneous, averaging between 2 and 4 degrees. PA in the sheep infarct is significantly elevated and allows the infarct zone to be differentiated from the rest of the myocardium (Figure $1 \mathrm{C}$-D). Both PA (Figure $2 \mathrm{~A}$ ) and Gd-DE uptake (Figure $2 \mathrm{~B}$ ) were significantly increased in the infarct zone of all the mouse hearts imaged. A PA threshold of 4 degrees robustly segmented the infarct zone (Figure 2C), and an excellent correlation $\left(R^{2}=0.94\right)$ was seen between percent infarct size by Gd-DE and tractographic PA (Figure 2D).

\section{Conclusions}

PA detects the loss of tract coherence in infarcted myocardium and robustly delineates myocardial infarcts in vivo. The use of DTI, and hence the tractographic PA, does not require exogenous contrast and can be performed in all patients regardless of renal function. The technique provides a complementary and valuable adjunct to $\mathrm{Gd}-\mathrm{DE}$.

\section{Funding}

R01HL093038. 




Figure 1 Tractograms color-coded by the propagation angle (PA). (A) PA is defined as the angle between two adjacent principal eigenvectors $\left(\hat{e}_{\mathrm{i},}, \hat{\mathrm{e}}_{\mathrm{ij}+1}\right)$ relative to a given myofiber. (B) Normal human heart viewed from the base, showing a low and homogenous PA. (C) Sheep heart with a large anteroseptal infarct. (C1) A low-pass PA value of 4 degrees delineates the normal myocardium and creates a void in the infarct. (C2) Conversely, a high-pass PA value of 4 degrees robustly delineates the infarcted myocardium.

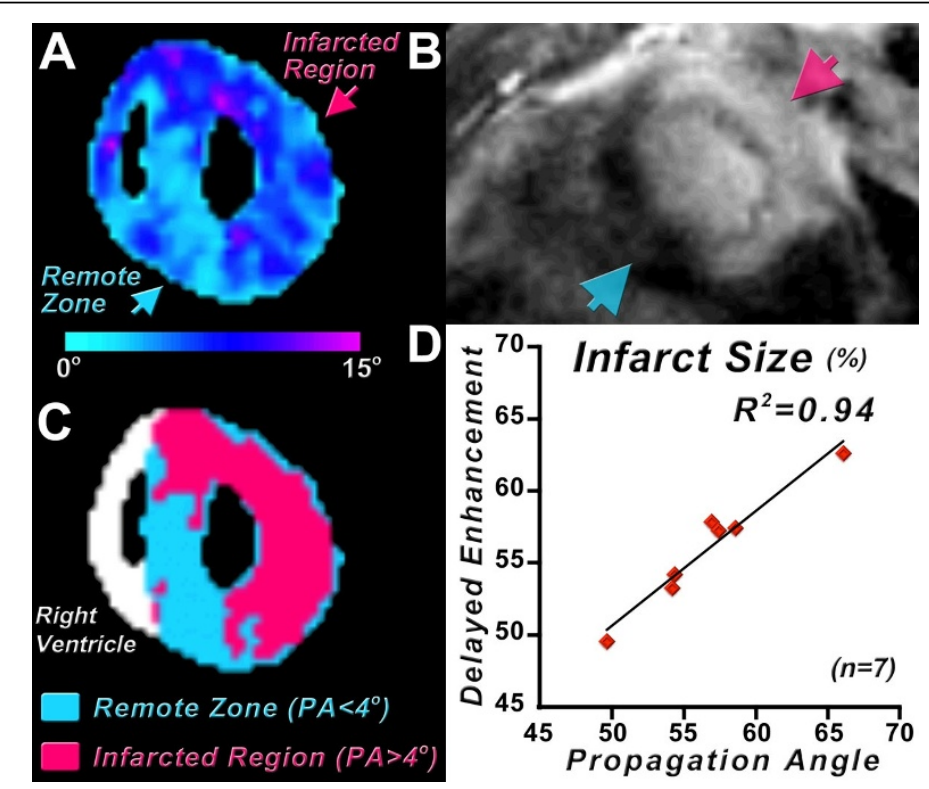

Figure 2 In vivo PA maps in infarcted mice. (A) PA map in a mouse with a large anterolateral infarct. (B) Delayed enhancement image at the corresponding level. It should be noted that the PA maps were acquired in mid-systole and the delayed enhancement images in mid-diastole. (C) Segmentation of the PA map using a threshold value of 4 degrees robustly segments normal from infarcted myocardium. (D) A high correlation $\left(\mathrm{R}^{2}=0.94\right)$ between infarct size calculated from the In vivo PA and infarct size measured by delayed gadolinium enhancement was obtained. 
Published: 30 January 2013

\section{References}

1. Kim R, et al:. NEJM 2000.

2. Mekkaoui C, et al:. ISMRM 2011.

doi:10.1186/1532-429X-15-S1-P2

Cite this article as: Mekkaoui et al:: Myocardial infarct delineation in vivo using diffusion tensor MRI and the tractographic propagation angle. Journal of Cardiovascular Magnetic Resonance 2013 15(Suppl 1):P2.

Submit your next manuscript to BioMed Central and take full advantage of:

- Convenient online submission

- Thorough peer review

- No space constraints or color figure charges

- Immediate publication on acceptance

- Inclusion in PubMed, CAS, Scopus and Google Scholar

- Research which is freely available for redistribution

Submit your manuscript at www.biomedcentral.com/submit 